\title{
T-cell-replete haploidentical bone marrow transplantation for X-linked severe combined immunodeficiency
}

Singapore Med J 2020; 61(5): 285-286 https://doi.org/10.11622/smedj.2018101

Dear Sir,

Without definitive therapy, severe combined immunodeficiency (SCID) is invariably fatal within the first two years of life. Allogeneic haematopoietic stem cell transplantation (HSCT) is curative; however, finding a human leucocyte antigen (HLA)-matched sibling donor is challenging. An unrelated donor graft is an alternative option, but it is limited by ethnic-associated disparities, high-costs and a long lag time to procurement. We share our experience in performing T-cell-replete haploidentical HSCT (hHSCT) in an infant with T-NK-B+SCID, leading to full immune reconstitution.

The infant was an only child of an ethnic Malay non-consanguineous couple. He was referred at ten months of age due to recurrent pneumonia, chronic diarrhoea and failure to thrive. His BCG (Bacille Calmette-Guérin vaccine) inoculation site was pustular and yielded acid-fast bacilli. Investigations showed markedly reduced T and NK cells with undetectable serum immunoglobulin levels (Table I). Genetic analysis revealed a heterozygous c.270-2A>T mutation in intron 2 of the IL2RG gene (also detected in his mother), confirming the diagnosis of X-linked SCID. Immediate measures included commencement of anti-tuberculosis therapy, as well as intensive nutritional and pulmonary rehabilitation.

Due to the urgency of his circumstances, a T-cell-replete 5/10 HLA-mismatched hHSCT was performed using his father's bone marrow as the stem cell source (nucleated and CD34+ cell dose of $6 \times 10^{8} / \mathrm{kg}$ and $1.7 \times 10^{6} / \mathrm{kg}$, respectively). The conditioning regimen consisted of thiotepa $5 \mathrm{mg} / \mathrm{kg}$ on Day 7, cyclophosphamide $30 \mathrm{mg} / \mathrm{kg}$ on Days 5 and 6, and fludarabine $30 \mathrm{mg} / \mathrm{m}^{2} / \mathrm{day}$ on Days 2-6 with no irradiation. This was a modification of the published Hopkins' hHSCT protocol. (1) Post transplantation, cyclophosphamide $50 \mathrm{mg} / \mathrm{kg} /$ dose was administered on Days +3 and +4 . A combination of oral mycophenolate mofetil and cyclosporin A was used as graft-versus-host-disease (GVHD) prophylaxis till Day +90 and Day +180 , respectively.

The infant achieved neutrophil engraftment by Day +10 and platelet engraftment on Day +11 . Lineage-specific chimerism analysis demonstrated full donor T-cell engraftment by Day +46. Control of GVHD was challenging, as he developed acute GVHD of the skin (Stage 3) and gut (Stage 1) on Day +12 and Day +53 , respectively. He also developed severe pneumonitis on Day +65 , which required mechanical ventilation for two days and bi-level positive airway pressure support for three weeks. Tracheal secretions were repeatedly sterile, while polymerase chain reaction screening for adenovirus, influenza and cytomegalovirus were negative.

Despite empirical broad-spectrum antibiotics, micafungin and cidofovir, the child's respiratory symptoms did not abate. A presumptive diagnosis of GVHD of the lungs was made. Pneumonitis resolved after three weeks of prednisolone and cyclophosphamide pulse therapy of $30 \mathrm{mg} / \mathrm{kg}$ on Days +105 and +106 . The patient was finally discharged from the ward on Day +149 with no re-admissions for illness. Currently, 48 months post hHSCT, he remains completely well with no evidence of GVHD since the HSCT, which was performed in April 2016.

Although the first HSCT to correct SCID was performed nearly 50 years ago, ${ }^{(2)}$ the optimal transplantation preparative therapy and even the need for any remains controversial. In this child's case, a reduced-intensity conditioning regimen was chosen to achieve a balance between ensuring engraftment while avoiding a flare-up of his underlying morbidities. T-cell-depleted hHSCT is currently the treatment of choice for infants with SCID who lack an HLA-matched donor. ${ }^{(3)}$ However, the high cost and expertise required to engineer the graft preclude this technique in countries with limited resources. Our case illustrates that hHSCT using unmanipulated T-cell-replete bone marrow is a feasible method to cure infants with SCID.

Table I. Immune profile prior to and after bone marrow transplantation (BMT).

\begin{tabular}{|c|c|c|c|c|}
\hline Immune profile & Normal range for age & Pre-BMT & Day + 280 & Day +783 \\
\hline Pan T cells (cells/ $\mu \mathrm{L})$ & $800-3,000$ & 445 & 2,756 & 6,675 \\
\hline CD4+ cells (cells $/ \mu \mathrm{L})$ & $600-1,900$ & 291 & 1,665 & 1,682 \\
\hline CD8+ cells (cells/ $\mu \mathrm{L})$ & $200-1,200$ & 81 & 1,087 & 4,080 \\
\hline NK cells (cells/ $\mu \mathrm{L}$ ) & $150-850$ & 127 & 321 & 161 \\
\hline Pan B cells (cells $/ \mu \mathrm{L})$ & $600-2,000$ & 2297 & 1,272 & 1,011 \\
\hline Immunoglobulin G (mg/dL) & $>400$ & $<6$ & 503 & 419 \\
\hline Immunoglobulin A (mg/dL) & $>23$ & $<23$ & 26 & 26 \\
\hline Immunoglobulin M (mg/dL) & $>17$ & $<17$ & 27 & 92 \\
\hline
\end{tabular}


Yours sincerely,

Hany $\underline{\text { Ariffin }}^{1}$, Kee Seang $\underline{\text { Chew }}^{1}$, Vida Jawin ${ }^{1}$, Surendran Thavagnanam ${ }^{2}$

${ }^{1}$ Paediatric Bone Marrow Transplant Unit, ²Division of Paediatric Respiratory Medicine, University of Malaya Medical Centre, Malaysia. hany@ummc.edu.my

\section{References}

1. Brodsky RA, Luznik L, Bolaños-Meade J, et al. Reduced intensity HLA-haploidentical BMT with post transplantation cyclophosphamide in nonmalignant hematologic diseases. Bone Marrow Transplant 2008; 42:523-7.

2. Meuwissen HJ, Gatti RA, Terasaki PI, Hong R, Good RA. Treatment of lymphopenic hypogammaglobulinemia and bone-marrow aplasia by transplantation of allogeneic marrow. Crucial role of histocompatibility matching. N Engl J Med 1969; 281:691-7.

3. Pai SY, Logan BR, Griffith LM, et al. Transplantation outcomes for severe combined immunodeficiency, 2000-2009. N Engl J Med 2014; 371:434-46. 